\title{
Equilíbrio e força de membros inferiores correlacionam-se com velocidade de marcha em pacientes com AVC: um estudo observacional
}

\author{
Balance and lower limb strength correlate with gait \\ speed in stroke patients: an observational study
}

\section{Caroline Camerin ${ }^{1}$ (]) \\ Rafael Dias Bittencourt ${ }^{2}$ (1) Luciano Palmeiro Rodrigues $^{3}$ (1)}

\begin{abstract}
1-2Universidade Federal do Rio Grande do Sul (Porto Alegre). Rio Grande do Sul, Brasil. camerincaroline@gmail.com, rafadb93@gmail.com ${ }^{3}$ Autor para correspondência. Universidade Federal do Rio Grande do Sul (Porto Alegre). Rio Grande do Sul, Brasil. Iucianopalmeiro@gmail.com
\end{abstract}

RESUMO | INTRODUÇÃO: A marcha e o equilíbrio são frequentemente comprometidos após lesões cerebrais como o Acidente Vascular Cerebral (AVC). OBJETIVO: Correlacionar a força dos membros inferiores e o equilíbrio em apoio unipodal no membro parético com a velocidade de marcha em pacientes com AVC. MÉTODOS: Estudo do tipo observacional transversal. Indivíduos de ambos os sexos com diagnóstico de AVC atendidos no Ambulatório de Neurovascular do Hospital de Clínicas de Porto Alegre, que conseguissem permanecer em ortostase por dois minutos sem auxílio e com marcha domiciliar com ou sem auxílio de dispositivos de marcha, foram avaliados quanto a força muscular de Msls, por meio do Teste de Sentar e Levantar em 30 segundos (TSL30s); avaliação do equilíbrio pelo Teste de Apoio Unipodal (AU) e da velocidade da marcha pelo Teste de Caminhada de 10 metros (TC10m) com auxílio de um acelerômetro fixado na região de $\mathrm{L} 5$. RESULTADOS: Dos trinta e quatro indivíduos avaliados, $67,6 \%$ eram do sexo feminino, com média de idade de $60,5( \pm 13,6)$ anos. A velocidade de marcha confortável média foi 1,01 \pm $0,31 \mathrm{~m} / \mathrm{s}$, a média no TSL30s foi $9 \pm 2,94$ repetições e o tempo médio de apoio no membro inferior acometido foi de 4,11 \pm 10,43 segundos. Houve correlação dos valores no TC10m com o tempo de permanência no lado acometido $(r=0,563 p<0,001)$ e com o número de repetições no TSL30s ( $r=0,667 p<0,001)$. CONCLUSÃO: Quanto maior a força muscular dos Msls e o equilíbrio em apoio unipodal sobre o membro inferior acometido, maior a velocidade de marcha nos pacientes com AVC.

PALAVRAS-CHAVE: Acidente vascular cerebral. Força muscular. Marcha. Equilíbrio. Velocidade de marcha.

\begin{abstract}
BACKGROUND: Gait and balance are often compromised after brain injuries such as stroke. OBJECTIVE: To correlate the lower limb muscle strength walking speed, and balance in unipedal support on a paretic limb with gait speed in stroke patients. METHODS: This study employed a correlational design. Individuals of both genders with a stroke diagnosis were seen at the Neurovascular Outpatient Clinic of the Hospital de Clínicas de Porto Alegre, Brazil, who were able to remain in orthostasis for two minutes without assistance and with walking at home with or without the aid of walking devices. The Sit-to-stand test for 30 seconds (STS 30s) was used to examine muscle strength of lower limbs; the balance was measured using the Unipedal Support Test (UST) and walking speed with 10 Meter Walk Test (10MWT) and used an accelerometer around the waist of the subject. RESULTS: A total of 34 subjects were evaluated, $67.6 \%$ female and mean age $60.5( \pm 13.6)$ years. The average comfortable walking speed was $1.01 \pm 0.31 \mathrm{~m} / \mathrm{s}$, the average in the STS 30s was $9 \pm$ 2.94 repetitions, and the average support time on the affected lower limb was $4.11 \pm 10.43$ seconds. In addition, there was a correlation between the values in the 10MWT and the length of stay on the affected side $(r=0.563 p<0.001)$ and with the number of repetitions in the STS 30s $(r=0.667 p<0.001)$. CONCLUSION: The greater the muscle strength of the lower limbs and the balance in unipedal support on the affected lower limb, the greater the gait speed in stroke patients.
\end{abstract}

KEYWORDS: Stroke. Muscle strength. Gait. Balance. Gait speed. 


\section{Introdução}

Após um AVC, muitos indivíduos apresentam consequências crônicas, prejudicando a função motora. Estas consequências variam de acordo com a área e a extensão da lesão, sendo comuns o aumento do tônus muscular (espasticidade), dos reflexos tendinosos (hiperreflexia), alterações no equilíbrio e diminuição de força muscular no hemicorpo acometido (hemiparesia). A hemiparesia é a disfunção motora mais presente nestes indivíduos. Esse acometimento prejudica a atividade muscular e a inibição recípro$\mathrm{ca}$, interferindo na velocidade de execução dos movimentos automáticos, como, por exemplo, a presença de alterações na velocidade da marcha em pacientes com AVC. ${ }^{1} \mathrm{O}$ desempenho da marcha e o equilíbrio são frequentemente comprometidos após lesões cerebrais como o AVC.?

A disfunção de marcha é uma das sequelas mais graves e incapacitantes após o AVC, atingindo cerca de $80 \%$ das pessoas acometidas no primeiro ano após a lesão, sendo que cerca de $30 \%$ destes indivíduos não recuperam esta capacidade totalmente com o passar do tempo. ${ }^{3.4} \mathrm{~A}$ diminuição na distribuição de carga no membro inferior acometido e o atraso nas reações de equilíbrio tornam a marcha hemiparética assimétrica, com alterações no comprimento do passo e na cadência, aumentando o tempo de apoio no membro não parético e o tempo de duplo apoio acarretando numa diminuição da sua velocidade. $\underline{5}$

A diminuição na velocidade de marcha é resultado de diversos fatores, incluindo a má recuperação motora e a diminuição de equilíbrio e da força muscular, gerando um efeito negativo sobre o nível de independência funcional do indivíduo. $\frac{6}{}$ Melhorar o padrão de marcha é considerado a principal meta no processo de reabilitação, pois sua limitação está intimamente relacionada com a realização de atividades de vida diária e qualidade de vida. $\frac{7}{}$ Tendo em vista que a força muscular tem importante influência na capacidade de caminhar, compreender a relação entre estas variáveis é de fundamental importância para a elaboração de intervenções eficazes na reabilitação da marcha destes pacientes. $\underline{3}$
Os estudos relacionam a velocidade de marcha ou a capacidade de marcha funcional com a força de grupos musculares específicos através da força isométrica máxima, utilizando a dinamometria. Outros relacionam o equilíbrio utilizando escalas como a Escala de Equilíbrio de Berg ou o Teste de Romberg com a velocidade de marcha. A correlação entre o equilíbrio através do Evaluation of the March and Oriented Balance for Performance (POMA) e a postura do paciente com AVC utilizando o Postural Assessment Scale after Stroke (PASS) e mobilidade funcional através do Test Timed Up and Go (TUG) já foram estudadas., ${ }^{3,-9} \mathrm{~A}$ relação entre a força muscular de ambos os membros inferiores através de um teste funcional como o Teste senta-levanta 30 segundos e o equilíbrio unipodal sobre o lado acometido no paciente de AVC com a velocidade de marcha cronometrada não foram estudados, tornando importante a realização deste estudo. Assim, o objetivo deste estudo é correlacionar a força dos membros inferiores e o equilíbrio em apoio unipodal no membro parético com a velocidade de marcha em pacientes com AVC.

\section{Metodologia}

Estudo observacional analítico, transversal com delineamento correlacional de amostragem não probabilística selecionada por conveniência, aprovado pelo Comitê de Ética do Hospital de Clínicas de Porto Alegre (número CAAE 67116917.7.3001.5327).

\section{População e Amostra}

O estudo foi realizado em pacientes com diagnóstico de AVC atendidos no Ambulatório Neurovascular do Hospital de Clínicas de Porto Alegre. Os critérios de inclusão foram: ter somente um episódio de AVC (isquêmico ou hemorrágico); conseguir permanecer em ortostase por dois minutos sem auxílio, com ou sem supervisão; possuir marcha domiciliar com ou sem auxílio de dispositivos de marcha; ter capacidade cognitiva para entender os testes. 
Os critérios de exclusão foram: presença de outras doenças neurológicas associadas; presença de lesões traumato-ortopédicas nos membros inferiores no último ano ou patologias respiratórias que afetassem o condicionamento cardiorrespiratório, para evitar fontes de viés.

\section{Procedimentos de Coleta e Análise}

Todos os participantes assinaram o Termo de Consentimento Livre e Esclarecido (TCLE) antes de participar do estudo, e a coleta dos dados ocorreu em apenas um dia, no período de fevereiro a julho de 2017. Os dados dos participantes como: sexo, idade, tipo de AVC, tempo de AVC e hemicorpo acometido foram coletados dos prontuários. Em seguida, os participantes realizavam o Teste de Sentar e Levantar em 30 segundos (TSL30s) para a mensuração da força muscular dos membros inferiores (Msls). Era solicitado que o participante levantasse da posição sentada no meio de uma cadeira com altura entre $43 \mathrm{~cm}$ e $45 \mathrm{~cm}$ com encosto, sem apoio de braços, com o tronco ereto e pés apoiados no chão por 30 segundos. O número de repetições que o indivíduo se levantar completamente de uma posição sentada no tempo máximo estabelecido reflete a força de membros inferiores. ${ }^{10,11}$ O Teste de Apoio Unipodal (AU) foi realizado no membro inferior acometido, para quantificar o equilíbrio. ${ }^{12,13}$ Os sujeitos ficaram com o pé do membro inferior acometido, levantado aproximadamente $5 \mathrm{~cm}$ do maléolo medial contralateral sem fazer contato do pé com o maléolo, mantendo os braços sobre o peito. Foi calculada a média do tempo das três tentativas. Se o indivíduo fosse incapaz de realizar o apoio unipodal sobre o lado acometido, a pontuação era de 0. Após, uma cinta na região lombar (na vértebra L5) foi utilizada para fixar o acelerômetro durante a realização do Teste de Caminhada de 10 metros (TC10m), que mensura a velocidade de marcha, em um corredor com 20 metros de comprimento. ${ }^{14}$ Os dados referentes aos parâmetros de marcha como cadência, comprimento do passo e duração de apoio no membro inferior acometido foram adquiridos por meio do Acelerômetro Loran Engineering ${ }^{\circledR}$.

\section{Análise Estatística}

O cálculo amostral foi realizado por meio do software G Power 3.1.7, utilizando a família de testes Z (teste de Correlação de Pearson - dependente). Assumiu-se uma correlação de 0,53 , um alfa de 0,05 , um tamanho de efeito de 0,70 e um poder de $80 \%$, a amostra mínima estimada para este estudo foi de 32 indivíduos.

A velocidade de marcha, o comprimento do passo, a cadência e a duração do apoio unipodal no membro inferior acometido foram consideradas variáveis dependentes. Já a força muscular nos Msls e o equilíbrio estático em AU foram consideradas variáveis independentes. Os dados de caracterização da amostra sobre gênero, tipo de AVC, lado acometido, foram apresentados como frequência simples e, a idade, tempo de AVC e de internação hospitalar, os valores do TC10m, do teste AU e do TSL30s foram apresentados em média e desvio padrão. Utilizou-se do coeficiente de correlação de Pearson para verificar a correlação entre as variáveis com distribuição normal. Todos os dados foram processados no software IBM SPSS 19, aceitando-se um nível de significância de $5 \%(p \leq 0,05)$.

\section{Resultados}

Um total de 34 sujeitos de ambos os sexos com histórico de apenas um AVC foram incluídos no estudo. As características da amostra estão descritas na Tabela 1. 
Tabela 1. Dados descritivos da amostra

\begin{tabular}{lcc}
\hline \multicolumn{1}{c}{ Variáveis } & n/Média & Desvio Padrão \\
\hline Gênero (Feminino/Masculino) & $23(67,6 \%) / 11(27,4 \%)$ & \\
Idade (anos) & 60,5 & \\
AVC (Isquêmico/Hemorrágico) & $29(85,2 \%) / 5(13,8 \%)$ & $\pm 375,9$ \\
Tempo de AVC (dias) & 71,5 & \\
Lado acometido (Direito/Esquerdo) & $9(58,8 \%) / 14(41,2 \%)$ & $\pm 7,2$ \\
Trombólise (\%) & 10 & $\pm 2,94$ \\
Tempo de internação hospitalar (dias) & 9 & $\pm 10,43$ \\
Força muscular TSL30s(repetições) & 4,11 & $\pm 0,31$ \\
Equilíbrio em AU (segundos) & 1,01 & \\
Velocidade de marcha (m/s) & & \\
\hline
\end{tabular}

TSL30s: Teste Senta-levanta 30 segundos; AU: Teste do Apoio Unipodal.

A partir dos dados coletados referentes à média do TC10m e considerando os valores normativos de gênero e idade, observou-se que os sujeitos avaliados apresentavam uma velocidade de marcha confortável diminuída. De acordo com o número médio de repetições dos pacientes no TSL30s, verificou-se que a força de membros inferiores estava diminuída e, com base no tempo médio de permanência em apoio unipodal sobre o membro inferior acometido o equilíbrio estava alterado, pois, não foram capazes de permanecer os 30 segundos, tempo máximo estabelecido pelo teste. As correlações entre essas variáveis estão descritas na Tabela 2.

Tabela 2. Correlação das variáveis: força muscular dos membros inferiores e equilíbrio com a velocidade de marcha

\begin{tabular}{llc}
\hline $\begin{array}{l}\text { Variáveis correlacionadas com a } \\
\text { velocidade de marcha }\end{array}$ & Medidas & $\begin{array}{c}\text { Teste de Correlação de } \\
\text { Pearson }\end{array}$ \\
\hline & TSL30s & $, 667,000$
\end{tabular}

Equilíbrio Estático no lado acometido

$\mathrm{AU}$

,563 001

TSL30s: Teste Senta-levanta 30 segundos; AU: Teste do Apoio Unipodal. Dados expressos em coeficiente de correlação (r) e nível de significância (p-valor) $(n=34)$.

Quando correlacionados os valores obtidos no TSL30s com os do TC10m, podemos observar que houve uma correlação positiva moderada entre essas variáveis, indicando que quanto maior o número de repetições de sentar e levantar realizadas pelos pacientes, maior a velocidade com o que os pacientes deambulavam confortavelmente (Gráfico 1). O tempo de permanência somente sobre o membro inferior acometido (AU) correlacionado com os valores do TC10m, demonstrou correlação positiva moderada, indicando que quanto maior o tempo de permanência em apoio unipodal no membro inferior acometido, maior era a velocidade com que o paciente caminhava Gráfico 2). 
Gráfico 1. Correlação de Pearson entre as variáveis força muscular de Msls e a velocidade de marcha confortável

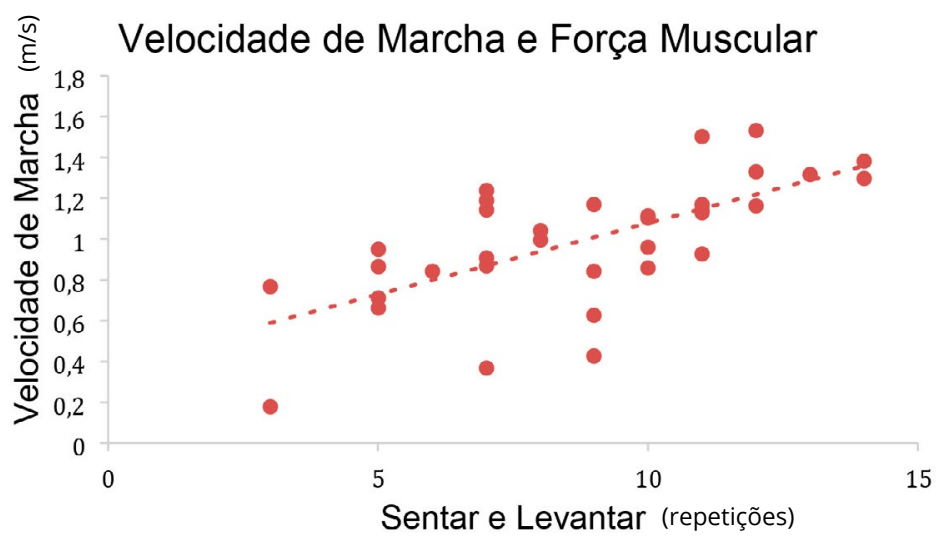

Gráfico 2. Correlação de Pearson entre as variáveis equilíbrio estático em apoio unipodal e a velocidade de marcha confortável

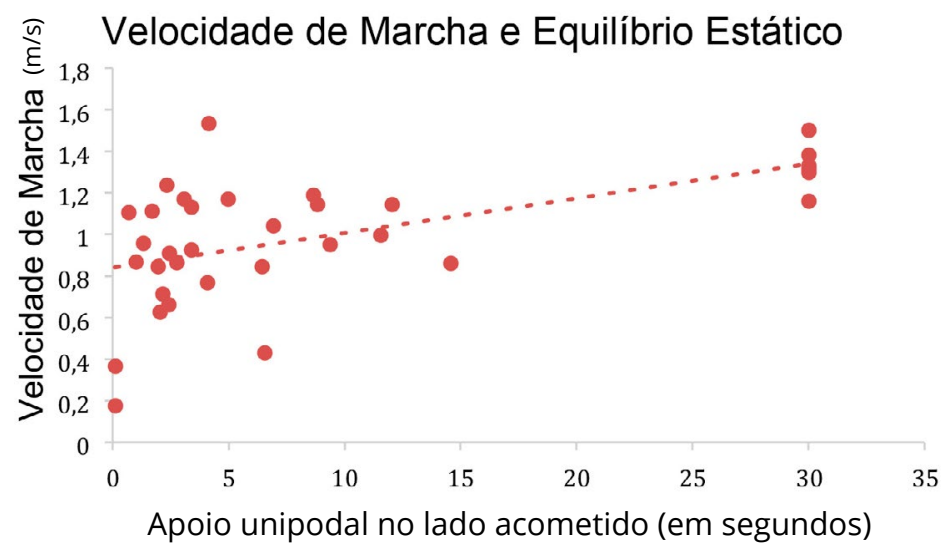

\section{Discussão}

No presente estudo, a variável força muscular foi mensurada de uma maneira mais funcional e o equilíbrio estático avaliado apenas no membro inferior acometido. Os dados obtidos sugerem que quanto maior a força muscular nos membros inferiores e maior equilíbrio sobre o membro inferior acometido, maior a velocidade de marcha dos pacientes após o AVC.

Após a lesão pelo AVC, aproximadamente $22 \%$ dos sobreviventes não conseguem deambular sem algum dispositivo de marcha, e desses, $26 \%$ se tornam dependentes nas AVDs. ${ }^{15} \mathrm{~A}$ marcha em pacientes de AVC é caracterizada por ser de baixa velocidade e possuir assimetrias, como o maior tempo da fase de apoio, menor tempo na de balanço e menor comprimento do passo. ${ }^{16} \mathrm{~A}$ marcha espontânea de indivíduos saudáveis tem velocidade média de 1,0 a 1,5 metros por segundo (m/s) enquanto a hemiparética está entre média de 0,45 e 0,75 m/s, caracterizando uma diminuição significativa. ${ }^{17} \mathrm{Em}$ nosso estudo, a média da velocidade de marcha confortável dos sujeitos foi abaixo do esperado para indivíduos nesta média de idade, a qual seria uma velocidade entre 1,15 m/s para muIheres e 1,27 para homens. ${ }^{18}$ Manter a velocidade de marcha normal é importante para a realização adequada de AVDs com independência, tornando estes pacientes mais ativos socialmente, e, por consequência, aumentando a sua qualidade de vida. ${ }^{17}$ Os pacientes avaliados após o AVC apresentaram tendência uma diminuição da velocidade de marcha. Neste estudo, não utilizamos uma medida de independência para verificar a interferência da velocidade de marcha com a capacidade de realizar as atividades de maneira independente. Esta é uma possível limitação do estudo. 
Além disso, os pacientes que tiveram uma velocidade de marcha mais rápida permaneceram maior tempo em apoio no membro inferior acometido. Lewek e colaboradores $^{19}$ citam que estes pacientes possuem uma fase de balanço do MI não acometido menor, pois o $\mathrm{Ml}$ acometido não tem condições de transferir e suportar o peso corporal durante sua fase de apoio. Portanto, pode-se inferir que, pacientes após

AVC tendem a dar mais passos devido à dificuldade em manter o membro inferior acometido em apoio unipodal durante a marcha, por déficits de equilíbrio e força muscular nos Msls, antecipando a fase de apoio do MI não acometido.

Taylor-Piliae et al..0 estudaram a interferência de diversas variáveis na adequada execução da marcha e encontraram que a diminuição da força muscular dos Msls é o maior contribuinte para a diminuição da velocidade de marcha. Já o estudo de Antunes et al. ${ }^{7}$ encontraram que, para uma marcha eficiente, também é necessário o controle do equilíbrio, já que a atividade muscular voluntária causa uma redistribuição na força interna e altera o centro de massa, causando uma instabilidade corporal. Além destes, um aspecto que não foi avaliado e que pode ter interferido nos resultados tanto do TSL30s quanto do TC10m é o aspecto sensorial, que se alterado pode influenciar na capacidade do paciente de realizar os testes de sentar e levantar e o de apoio unipodal.

A média aferida no TSL30s mostrou diminuição da força muscular de Msls, já que nesta média de idade se espera que o sujeito consiga sentar e levan$\operatorname{tar} 14$ vezes se for mulher e 16 se for homens. ${ }^{10}$ Corroborando com esses números, um estudo de Dorsch, Ada, Canning ${ }^{8}$ mediu a força muscular dos membros inferiores de pacientes pós AVC e comparou com as medidas de controle para a mesma idade e sexo, encontrando que a força do membro inferior parético foi em equivalente a $48 \%$ ao do grupo controle, e a do membro inferior saudável foi equivalente a $66 \%$ ao do grupo controle. Ainda discutem que essa diferença de força muscular em ambos os membros inferiores e não somente no parético se deve ao desuso dessa musculatura pela diminuição da realização de suas atividades após a lesão.
Uma revisão sistemática²1 analisou 21 estudos que correlacionaram a força muscular de membros inferiores com a velocidade de marcha em pacientes que sofreram AVC e todos os estudos apresentaram correlação positiva de moderada a forte entre as variáveis. Porém, os estudos utilizados na revisão avaliaram a força muscular de grupos musculares isolados. Nosso estudo optou pela utilização do TSL30s para mensurar a força muscular dos Msls, uma vez que é um teste de fácil replicação na prática clínica e pode ser utilizado como exercício na reabilitação dessa população, sendo este um dos diferenciais do estudo, o fato de ter utilizado medidas de uso clínico na verificação das variáveis estudadas. A atividade de sentar e levantar pode ser desafiadora para pacientes com AVC, já que a dificuldade na distribuição de peso corporal em ambos os Msls, pode fazer com que esta atividade seja mal executada. 22 Este fator pode explicar o número reduzido de repetições realizadas durante o teste, caracterizando a diminuição de força muscular, de acordo com o teste, o que também justificaria a dificuldade na sua realização. Uma revisão $\underline{23}$ de 29 artigos que avaliaram a atividade de sentar e levantar em pacientes com AVC demonstrou que estes pacientes demoram mais para executar esta tarefa e tendem a compensar a falta de força muscular nos membros inferiores com movimentos do tronco. Por isso, o ganho da força muscular dos Msls se torna essencial no processo de reabilitação, para que se garanta a funcionalidade e independência desses pacientes.

Além disso, a velocidade de marcha considerada adequada depende também da força muscular dos Msls, o que pode ser confirmado pela correlação positiva moderada encontrada neste estudo. Carvalho e colaboradores 24 analisaram a força muscular e a velocidade de marcha em pacientes com AVC e compararam com pacientes saudáveis, encontrando que pacientes que os sujeitos com AVC caminharam apenas $77 \%$ do e previsto quando comparado ao grupo saudável e a força muscular do membro inferior afetado equivalia a $61 \%$ da força do membro inferior de um sujeito saudável quando equiparado. E quando correlacionaram as duas variáveis, encontraram que quem possuía menor força muscular, deambulava com menor velocidade, corroborando com os achados neste estudo, confirmando a correlação encontrada neste estudo de que quanto maior a força muscular de Msls, maior a velocidade de marcha dos pacientes com AVC. 
No equilíbrio estático, um indivíduo deveria conseguir permanecer 30 segundos em apoio unipodal12, portanto, o tempo médio alcançado pelos pacientes analisados em AU no hemicorpo acometido os caracteriza com alteração do equilíbrio estático. $O$ equilíbrio é importante para a realização de atividades funcionais, como caminhar, sentar e levantar, por isso, as diminuições prejudicam o desempenho desses pacientes nas suas atividades de vida diária. $\underline{25}$ Nosso estudo utilizou o AU como medida de equilíbrio estático e, mesmo a marcha sendo uma atividade dinâmica, foi encontrada correlação positiva com o equilíbrio em apoio unipodal. Este fato pode ser explicado devido à dificuldade que o paciente com AVC apresenta de realizar a transferência e suporte de peso para o hemicorpo acometido, prejudicando assim a fase de apoio na marcha, com consequente redução do comprimento de passo e, portanto, uma diminuição da velocidade de marcha.

Foi observado ainda um elevado desvio padrão no tempo de AVC. Tal achado deve-se a não definição nos critérios de inclusão de um tempo máximo de acometimento, tendo sido avaliados sujeitos com tempo de lesão que variavam de meses a anos, tornando a amostra menos homogênea. Mesmo assim, os achados deste estudo foram relevantes, porém as limitações do estudo, como a não padronização da posição dos pés durante a realização do TSL30s, pode ter levado o paciente a escolher levantar com membro inferior não acometido atrás, o que pode influenciar a performance do teste. Além disso, a alteração do apoio sobre o lado acometido e as alterações na marcha podem estar relacionadas aos aspectos sensoriais e de tônus muscular, tendo sido necessário que os pacientes selecionados para a amostra tivessem sido previamente avaliados e caracterizados quanto a sensibilidade e tonicidade em Msls. Uma amostra mais homogênea quanto a fase após AVC os pacientes se encontravam, talvez possibilitasse que os pacientes apresentassem características mais homogêneas. Estas limitações não permitem que sejam extrapolados alguns dos resultados deste estudo para a população em geral acometida pelo AVC.

A recuperação da capacidade de marcha é um dos principais objetivos da fisioterapia na reabilitação após o AVC e pode ser acompanhada pela medida da velocidade de marcha. $\underline{26} \mathrm{O}$ conhecimento de como o déficit de força muscular e outros componentes se relacionam com a marcha pode ser útil para os fisioterapeutas na avaliação e no planejamento de suas intervenções. ${ }^{24}$
Ao analisarmos as correlações encontradas entre as variáveis estudadas, sugere-se que o aumento da velocidade de marcha em pacientes hemiparéticos após AVC possa ser um objetivo de tratamento a ser alcançado através da melhora do apoio unipodal no lado acometido e da força muscular dos Msls.

\section{Conclusão}

O presente estudo mostrou que pacientes com AVC possuem diminuição da força muscular dos Msls, do equilíbrio estático e da velocidade de marcha quando comparados a sujeitos saudáveis. Estabeleceu-se correlação positiva entre a força muscular de Msls e equilíbrio estático no membro inferior acometido com a velocidade de marcha. Portanto, os pacientes que possuem menor força muscular dos Msls e alteração do equilíbrio estático apresentam diminuição da velocidade de marcha.

\section{Contribuições dos autores}

Rodrigues LP, Camerin CER participaram da concepção, delineamento, coleta e análise estatística dos dados, interpretação dos resultados e redação do artigo científico do artigo científico. Bittencourt RD participou da coleta e análise dos dados e interpretação dos resultados.

\section{Conflitos de interesses}

Nenhum conflito financeiro, legal ou político envolvendo terceiros (governo, empresas e fundações privadas, etc.) foi declarado para nenhum aspecto do trabalho submetido (incluindo, mas não se limitando a subvenções e financiamentos, participação em conselho consultivo, desenho de estudo, preparação de manuscrito, análise estatística, etc.).

\section{Referências}

1. Luvizutto GJ, Gameiro MO. Efeito da espasticidade sobre os padrões lineares de marcha em hemiparéticos. Fisioter Movi. 2011;24(4):705-12. https://doi.org/10.1590/S0103$\underline{51502011000400015}$

2. Tally Z, Boetefuer L, Kauk C, Perez G, Schrand L, Hoder J. The efficacy of treadmill training on balance dysfunction in individuals with chronic stroke: a systematic review, Top Stroke Rehabili. 2017;24(7):539-46. https://doi.org/10.1080/10749357.2 $\underline{017.1345445}$ 
3. Moriello C, Finch L, Mayo NE. Relationship between muscle strength and functional walking capacity among people with stroke. J Rehabil Res Dev. 2011;48(3):267-76. http://dx.doi. org/10.1682/jrrd.2010.04.0066

4. Seo JP, Lee MY, Kwon HY, Jang SH. Delayed gait recovery in a stroke patient. Neural Regen Res. 2013;5(8):1514-8. http://dx.doi. org/10.3969/j.issn.1673-5374.2013.16.008

5. Balaban B, Tok F. Gait Disturbances in Patients With Stroke. Pm\&r. 2014;6(7):635-42. http://dx.doi.org/10.1016/j. pmrj.2013.12.017

6. Beaman CB, Peterson CL, Neptune RR, Kautz SA. Differences in selfselected and fastest-comfortable walking in post-stroke hemiparetic persons. Gait Posture. 2010;31(3):311-16. http:// dx.doi.org/10.1016/j.gaitpost.2009.11.011

7. Antunes JE, Justo FHO, Justo AFO, Ramos GC, Prudente COM. Influência do controle postural e equilíbrio na marcha de pacientes com sequela de acidente vascular cerebral. Rev Fisioter S Fun [Internet]. 2016;5(1):30-41. http://www.repositorio.ufc.br/ handle/riufc/19343

8. Dorsch S, Ada L, Canning CG. Lower Limb Strength Is Significantly Impaired in All Muscle Groups in Ambulatory People with Chronic Stroke: a cross-sectional study. Arch Phys Med Rehabil. 2016;97(4):522-7. http://dx.doi.org/10.1016/j. apmr.2015.10.106

9. Warren M, Ganley KJ, Pohl PS. The association between social participation and lower extremity muscle strength, balance, and gait speed in US adults. Prev Med. 2016;4:142-47. http://dx.doi. org/10.1016/j.pmedr.2016.06.005

10. Jones CJ, Rikli RE, Beam WC. A 30-s chair-stand test as a measure of lower body strength in community-residing older adults. Res Q Exerc Sport. 1999;70(2):113-9. http://dx.doi.org/10.1 080/02701367.1999.10608028

11. Rikli RE, Jones CL. Development and validation of a functional fitness test for a community-residing adults. J Aging Phys Act. 1999;7(2):129-61. https://doi.org/10.1123/japa.7.2.129

12. Bohannon RW, Larkin PA, Cook AC, Gear J, Singer J. Decrease in timed balance test scores with aging. Phys Ther. 1984;64(7):106770. http://dx.doi.org/10.1093/ptj/64.7.1067

13. Cho BL, Scarpace D, Alexander NB. Tests of stepping as indicators of mobility, balance, and fall risk in balance impaired older adults. J Am Geriatr Soc. 2004;52(7):1168-73. http://dx.doi: 10.1111/j.1532-5415.2004.52317.x

14. Peters DM, Fritz SL, Krotish DE. Assessing the reliability and validity of a shorter walk test compared with the 10-meter walk test for measurements of gait speed in healthy, older adults. J Geriatr Phys Ther. 2013;36(1):24-30. http://dx.doi.org/10.1519/ jpt.0b013e318248e20d
15. Helgason CM, Wolf PA. American Heart Association Prevention Conference IV: prevention and rehabilitation of stroke. Circulation. 1997;96(2):701-7. http://dx.doi.org/10.1161/01.cir.96.2.701

16. Beinoitti F, Correia N, Christofoletti G, Borges G. Use of hippotherapy in gait training for hemiparetic post-stroke. Arq Neuro-psiquiatr. 2010;68(6):908-13. http://dx.doi.org/10.1590/ s0004-282x2010000600015

17. Khanittanuphong $P$, Tipchatyotin S. Correlation of the gait speed with the quality of life and the quality of life classified according to speed-based community ambulation in Thai stroke survivors. Neurorehabil Neural Repair. 2017;41(1):135-41. http:// dx.doi.org/10.3233/nre-171465

18. Oberg T, Karszinia A, Oberg K. Basic gait parameters: Reference data for normal subjects, $10-79$ years of age. J Rehabil Res Dev. 1993;30(2):210-23. Citado em: PMID: 8035350.

19. Lewek MD, Bradley CE, Wutzke CJ, Zinder SM. The relationship between spatiotemporal gait asymmetry and balance in individuals with chronic stroke. J Biomech. 2014;30(1):31-36. http://dx.doi.org/10.1123/jab.2012-0208

20. Taylor-Piliae RE, Daniel L, Hepworth JT, Coull BM. Predictors of gait velocity among community-dwelling stroke survivors. Gait Posture. 2012;35(3):395-99. http://dx.doi.org/10.1016/j. gaitpost.2011.10.358

21. Mentiplay BF, Adair B, Bower KJ, Williams G, Tole G, Clark RA. Associations between lower limb strength and gait velocity following stroke: a systematic review. Brain Inj. 2014;29(4):409-22. http://dx.doi.org/10.3109/02699052.2014.995231

22. Lecours J, Nadeau S, Gravel D, Teixera-Salmela L. Interactions between foot placement, trunk frontal position, weightbearing and knee moment asymmetry at seat-off during rising from a chair in healthy controls and persons with hemiparesis. J Rehabil Med. 2008;40(3):200-7. http://dx.doi.org/10.2340/16501977-0155

23. Boukadida A, Piotte F, Dehail P, Nadeau S. Determinants of sit-to-stand tasks in individuals with hemiparesis post stroke: a review. Ann. Phys. Rehabil. 2015;58(3):167-72. http://dx.doi. org/10.1016/j.rehab.2015.04.007

24. Carvalho C, Sunnerhagen KS, Willén C. Walking Performance and Muscle Strength in the Later Stage Poststroke: a nonlinear relationship. Arch Phys Med Rehabil. 2013;94(5):845-50. http:// dx.doi.org/10.1016/j.apmr.2012.11.034

25. Carr JH, Edinburgh RS. Neurological rehabilitation: optimizing motor performance. Physiother Can. 2010;67(2):215-16. https:// doi.org/10.3138/ptc.67.2.rev2

26. Eng JJ, Tang P. Gait training strategies to optimize walking ability in people with stroke: a synthesis of the evidence. Expert Rev Neurother. 2007;7(10):1417-36. http://dx.doi. org/10.1586/14737175.7.10.1417 